\title{
Depression in paediatric chronic fatigue syndrome
}

\author{
Helen Bould, ${ }^{1}$ Simon M Collin, ${ }^{1}$ Glyn Lewis, ${ }^{1}$ Katharine Rimes, ${ }^{2}$ Esther Crawley ${ }^{1}$
}

${ }^{1}$ School of Social and Community Medicine, University of Bristol, Bristol, UK ${ }^{2}$ Institute of Psychiatry, King's College London, London, UK

\section{Correspondence to}

Dr Esther Crawley,

Centre for Child and

Adolescent Health,

School for Social and

Community Medicine

Oakfield House, Oakfield

Grove, Bristol BS8 2BN, UK;

esther.crawley@bristol.ac.uk

Received 14 November 2012

Revised 3 March 2013

Accepted 24 March 2013

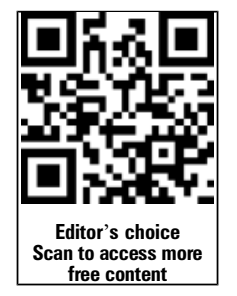

To cite: Bould $\mathrm{H}$, Collin SM, Lewis $\mathrm{G}$, et al. Arch Dis Child 2013;98:425-428.

\begin{abstract}
Objective To describe the prevalence of depression in children with chronic fatigue syndrome (CFS)/myalgic encephalomyelitis (ME) and investigate the relationship between depression in CFS/ME and clinical symptoms such as fatigue, disability, pain and school attendance. Design Cross-sectional survey data using the Hospital Anxiety and Depression Scale (HADS) collected at assessment.
\end{abstract}

Setting Specialist paediatric CFS/ME service in the South West.

Patients Children aged 12-18 years with CFS/ME.

Main outcome measure Depression was defined as scoring $>9$ on the HADS depression scale.

Results 542 subjects had complete data for the HADS and $29 \%(156 / 542)(95 \% \mathrm{Cl} 25 \%$ to $33 \%)$ had depression. In a univariable analysis, female sex, poorer school attendance, and higher levels of fatigue, disability, pain, and anxiety were associated with higher odds of depression. Age of child and duration of illness were not associated with depression. In a multivariable analysis, the factors most strongly associated with depression were disability, with higher scores on the physical function subscale of the 36 item Short Form (SF-36).

Conclusions Depression is commonly comorbid with CFS/ME, much more common than in the general population, and is associated with markers of disease severity. It is important to screen for, identify and treat depression in this population.

\section{INTRODUCTION}

Chronic fatigue syndrome (CFS)/ME is a disabling condition with a prevalence of between $0.2 \%$ and $2 \%{ }^{1-4}$ More than half of children and young people with CFS/ME are bed-bound at some stage, and they miss one academic year of schooling on average. ${ }^{5}$ In the UK, CFS/ME is defined as 3 months of disabling fatigue plus at least one additional symptom persisting after routine tests and investigations have failed to identify an obvious underlying cause. ${ }^{67}$

Depression is one of the most common emotional disorders in children and adolescents, with a prevalence of $2 \%-3 \% .^{8}$ Several small case-control studies describe a higher rate of depression in adolescents with CFS/ME than in healthy controls ${ }^{9-11}$ and higher depression scores than seen in the general population. ${ }^{12}$ Rates of depression are higher in adolescents with CFS/ME than in adolescents with other chronic illnesses such as juvenile idiopathic arthritis and migraine. ${ }^{10}{ }^{13}$ However, these studies were too small to determine precisely the prevalence of depression in CFS/ME or to examine the association of depression with patient characteristics that may predict depression, such as length of illness. For example, if depression was a consequence of the disabling nature of $\mathrm{CFS} / \mathrm{ME}$, one might expect a longer

\section{What is already known}

Small case-control studies show higher rates of depression in adolescents with chronic fatigue syndrome (CFS)/myalgic encephalomyelitis (ME) than in controls.

\section{What this study adds}

Rates of depression are ten times higher in adolescents with chronic fatigue syndrome (CFS)/myalgic encephalomyelitis (ME) than in the general population.

- Adolescents with CFS/ME who are depressed have higher levels of disability, pain, fatigue and anxiety than those who are not depressed.

- Clinicians should screen for and treat depression in adolescents with CFS/ME.

duration of illness to be associated with a higher prevalence of depression.

The aims of this study were: first, to describe the prevalence of depression in children with $\mathrm{CFS} / \mathrm{ME}$ presenting to a specialist CFS/ME clinic to help in the design of services; and, second, to investigate the association of depression in paediatric CFS/ME with patient characteristics such as age and sex, and with other clinical features such as fatigue, disability and pain, and school attendance. We hypothesised that length of time to diagnoses would be associated with depression in this cohort.

\section{METHODS \\ Population}

The Bath specialist paediatric CFS/ME service at the Royal National Hospital for Rheumatic Diseases covers a region in the south west of England with a population of over 400000 children aged 5-19 years. It also receives referrals from parts of England without paediatric CFS/ME services. Children are referred from paediatric services, general practices and schools. The service offers evidence-based treatment for severe, moderate and mild CFS/ME according to National Institute for Clinical Excellence (NICE) guidelines. ${ }^{6}$ All children complete screening inventories prior to assessment to identify comorbid depression and anxiety. Data were collected prospectively on all children assessed by the service between 1 January 2005 and 31 December 2011. We analysed data from those who were between the ages of 12 and 18 years at the time of assessment. Only children with a primary diagnosis of CFS/ME according to NICE 
criteria $^{6}$ were included in the analysis; children in whom fatigue was considered to be secondary to another diagnosis (including depression) were excluded. Children for whom depression was considered to be the primary problem causing fatigue and disability were referred to Child and Adolescent Mental Health Services for assessment and treatment.

\section{Inventories and scores}

All children were asked to complete questionnaires at assessment. The Hospital Anxiety and Depression Scale (HADS) was used to detect symptoms of depression ${ }^{14}{ }^{15}$ in children aged $\geq 12$ years. The HADS is a widely used self-report measure based on feelings and behaviour during the previous week. It comprises 14 items, seven for depression and seven for anxiety, each scored 0-3, leading to a maximum score of 21 for each subscale. The HADS has been recommended as a screening tool in $\mathrm{CFS}^{16}$ and has been shown to be reliable and valid in adolescents. ${ }^{14}$ We used the depression score both as a continuous variable and with a score $>9$ indicating probable depression (which we will refer to as depression). Anxiety was rated using the anxiety subscale of the HADS as a continuous variable. The Spence Children's Anxiety Scale (SCAS) was also completed as a measure of anxiety. ${ }^{17}$ The 11-item Chalder Fatigue Scale ${ }^{18}$ was scored using the $0-3$ method for scoring each question ( 0 for 'less than usual', 1 for 'no more than usual', 2 for 'more than usual' and 3 for 'much more than usual'). The 10-item physical function subscale of the Rand 36-Item Short Form (SF-36) Health Survey ${ }^{19}$ was used to assess physical function. Questions are scored 0 ('no, not limited at all'), 5 ('yes, limited a little') and 10 ('yes, limited a lot') so that the most disabled children score 0 while those with the least disability score 100. All questionnaire scores were coded as missing if more than one question was unanswered. If one question was unanswered the total score was rescaled using the mean response for the non-missing items. Pain was measured using a Visual Analogue Pain Rating Scale. Time-to-assessment was calculated as self-reported time from symptom onset to clinical assessment in the specialist clinic. Time at school was measured using a single self-completed questionnaire item on school attendance over the last term.

\section{Statistical analysis}

We used $\chi^{2}$ and Student $t$ tests to compare characteristics of the group of children for whom questionnaire data were complete with those for whom data were missing. We used logistic regression to investigate associations of fatigue, anxiety, physical function, pain, school attendance and time-to-assessment with depression. All analyses were restricted to children with no missing data in any of the variables investigated. Children with CFS/ME tend to mark the HADS item 'I feel slowed down' with the highest possible score ('Nearly all the time'). In order to assess whether this disproportionate response would affect the proportion of children with comorbid depression, we performed a sensitivity analysis in which we removed the item 'I feel slowed down' from the HADS depression subscale, replacing the score for that item with the mean score for the other items.

\section{RESULTS}

\section{Numbers of patients and missing data}

Between 1 January 2005 and 31 December 2011, a total of 674 children and young people between the ages of 12 and 18 years were seen in the service and met NICE diagnostic criteria for CFS/ME. Table 1 describes 542 (80.4\%) young people who completed the HADS and who were included in the analyses compared with those who did not complete the HADS. Nearly half (63/132) did not complete any inventories. Sex, school attendance, time-to-assessment, disability, pain, fatigue and anxiety (measured using the SCAS) were similar between the two groups. Those with completed HADS were slightly older (14.8 vs 13.9 years; $\mathrm{p}<0.001)$.

\section{Prevalence of depression in children with CFS/ME}

The mean HADS depression score was 7.6 (SD 3.8) in those with CFS/ME. The mean HADS depression score was lower in boys $(6.7$ (SD 3.5)) than in girls $(7.9(\mathrm{SD} 3.9))(\mathrm{p}<0.001)$ with $\mathrm{CFS} / \mathrm{ME}$. The proportion of children and adolescents with CFS/ME who had depression (HADS depression score $>9$ ) was $28.8 \%(156 / 542)(95 \%$ CI $25 \%$ to $33 \%)$. The proportion with a HADS depression score $\geq 8$ was $49.1 \%$ (266/542). Sensitivity analysis showed that the proportion of children with depression remained similar $(25.5 \%(138 / 542))$ when the HADS depression score was calculated excluding the item 'I feel slowed down', and this proportion was not different from that calculated using the full HADS depression subscale $(p=0.20)$.

\section{Correlates of depression}

Of the 542 children with complete HADS measurements, 404 children had complete data on all variables. Table 2 shows the ORs for associations of school attendance, fatigue, anxiety, pain and physical function with depression (HADS score >9). After adjustment for other variables, depression was associated with worse physical function (OR 0.77 (95\% CI 0.67 to 0.88 ) per 10-point increment on the SF-36 physical function subscale, $\mathrm{p}<0.001)$. The associations with pain (OR 1.13 (95\% CI 1.03

Table 1 Characteristics of children and young people aged 12 with CFS/ME who provided information on preassessment HADS inventory compared with those who did not provide information

\begin{tabular}{|c|c|c|c|c|c|c|}
\hline & \multicolumn{2}{|c|}{ With HADS } & \multicolumn{2}{|c|}{ Without HADS } & \multirow[b]{2}{*}{ Mean difference $(95 \% \mathrm{Cl})$} & \multirow[b]{2}{*}{$\mathrm{p}$ Value } \\
\hline & $\mathrm{N}$ & & $\mathrm{N}$ & & & \\
\hline Age in years: mean (SD) & 542 & $14.8(1.5)$ & 132 & $13.9(1.6)$ & $-0.84(-1.13,-0.54)$ & $<0.001$ \\
\hline Female sex $(\%)$ & 542 & $404(74.5 \%)$ & 132 & $94(71.2 \%)$ & Not applicable & 0.44 \\
\hline School attendance less than $40 \%$ & 520 & $214(41.2 \%)$ & 71 & $32(45.1 \%)$ & Not applicable & 0.53 \\
\hline Time to assessment (months): mean (SD) & 490 & $23.1(25.5)$ & 120 & $27.1(23.4)$ & $3.97(-1.05,8.99)$ & 0.12 \\
\hline Disability (SF-36): mean (SD) & 520 & $51.0(24.4)$ & 74 & $48.0(3.1)$ & $-3.07(-9.10,2.97)$ & 0.32 \\
\hline Chalder Fatigue Score: mean (SD) & 526 & $24.9(4.9)$ & 69 & $23.5(5.1)$ & $-1.33(-2.56,-0.03)$ & 0.10 \\
\hline SCAS: mean (SD) & 490 & $32.0(18.2)$ & 69 & $27.5(20.7)$ & $-4.51(-9.19,0.18)$ & 0.06 \\
\hline Visual Analogue Pain: mean (SD) & 491 & $47.4(28.5)$ & 66 & $48.7(30.1)$ & $1.26(-6.13,8.66)$ & 0.74 \\
\hline
\end{tabular}

CFS/ME, chronic fatigue syndrome/myalgic encephalomyelitis; HADS, Hospital Anxiety and Depression Scale; SCAS, Spence Children's Anxiety Scale; SF-36, 36-Item Short Form. 
Table 2 ORs for relationships between probable depression (HADS >9) and sex, age, physical function, anxiety, pain, school attendance, fatigue score and clinic latency $(\mathrm{N}=404)$

\begin{tabular}{|c|c|c|}
\hline & OR $(95 \% \mathrm{Cl}), \mathrm{p}$ value & Adjusted OR $(95 \% \mathrm{Cl}), \mathrm{p}$ value* \\
\hline Age (years) & 0.90 (0.78 to 1.04$), p=0.17$ & $1.00(0.84$ to 1.18$), p=0.96$ \\
\hline Female sex & $2.05(1.20$ to 3.51$), p=0.01$ & $1.82(1.00$ to 3.31$), p=0.05$ \\
\hline Fatigue (per 3-point increment on Chalder Fatigue Scale)† & 1.75 (1.44 to 2.14$), p<0.0001$ & $1.26(1.02$ to 1.57$), p=0.04$ \\
\hline Physical function (per 10-point increment on SF-36 physical function subscale) $\dagger$ & $0.67(0.60$ to 0.75$), p<0.0001$ & 0.77 (0.67 to 0.88$), p<0.001$ \\
\hline Pain (per $10 \mathrm{~mm}$ increment on Visual Analogue Pain Rating Scale)t & 1.25 (1.15 to 1.36$), \mathrm{p}<0.0001$ & $1.13(1.03$ to 1.25$), p=0.01$ \\
\hline School attendance of $40 \%$ or more & $0.36(0.23$ to 0.56$), p<0.0001$ & 0.75 (0.44 to 1.28$), p=0.28$ \\
\hline Duration of illness (months) & 1.00 (0.99 to 1.01$), p=0.80$ & 0.99 (0.98 to 1.00$), p=0.21$ \\
\hline Anxiety (per 2-point increment on HADS anxiety subscale) $\dagger$ & 1.40 (1.26 to 1.57$), p<0.0001$ & Not in model \\
\hline
\end{tabular}

${ }^{*}$ Multivariable analyses, adjusted for all variables in table except HADS anxiety.

tChalder Fatigue, HADS anxiety, SF-36 physical function and Visual Analogue Pain measures were rescaled to range from 0 to 10 . Original scales are: Chalder 0-33; HADS Anxiety 0-21; SF-36 0-100; Visual Analogue Pain 0-100.

HADS, Hospital Anxiety and Depression Scale; SF-36, 36-Item Short Form.

to 1.25$)$ per $10 \mathrm{~mm}$ increment on the Visual Analogue Pain Rating Scale, $\mathrm{p}<0.01$ ) and fatigue (OR 1.26 (95\% CI 1.02 to 1.57) per 3-point increment on the Chalder Fatigue Scale, $\mathrm{p}=0.04$ ) were attenuated but remained present.

Following adjustment, there was no evidence for an association between depression and school attendance or depression and length of illness prior to assessment.

\section{DISCUSSION}

The proportion of young people with CFS/ME meeting the criteria for depression on the HADS is between 8- and 14-fold higher than in the same age group in the general population. In a sample of 248 British school children aged 12-16, the prevalence of HADS depression score $>9$ was $2 \%{ }^{14}$ compared with $28.8 \%$ in our study. In a Swedish population sample of 585 13-23-year-olds, the prevalence of HADS depression score of $\geq 8$ was $4.0 \%$ (by phone interview) and $6.0 \%$ (by postal questionnaire $)^{20}$ compared with $49.1 \%$ in our study. Mean HADS depression scores in the Swedish study ranged from 1.8 (SD 2.3 ) in 13-15-year-old boys in postal questionnaires to 3.8 (3.3) in 16-19-year-old male subjects in postal questionnaires; ${ }^{20}$ the mean score in the British study was $3.4 ;^{14}$ these scores are much lower than our mean scores of 6.7 (3.5) for boys, 7.9 (3.9) for girls and 7.6 (3.8) overall. Children with CFS/ME who were depressed had higher levels of disability and anxiety compared with those who were not depressed. Depression was also associated with being female, being more fatigued and pain. There did not appear to be any association between depression and length of illness prior to first assessment. Following adjustment, there was no evidence of an association between depression and lower levels of school attendance.

\section{Strengths and limitations}

This is the first study of depression in a large cohort of children with CFS/ME presenting to specialist services. Our sample size has enabled us to compare those with and without comorbid depression in terms of their levels of disability, pain, fatigue, school attendance and anxiety. However, the study does have a number of limitations. First, the children in our study were referred to a specialist service, and it is unclear whether these results are generalisable to the wider CFS/ME paediatric population. Although children with depression and CFS/ME may be more likely to be referred to a specialist service than those with $\mathrm{CFS} / \mathrm{ME}$ alone, studies suggest that children with CFS/ME seen in non-specialist settings also have high rates of mental health difficulties, particularly emotional problems. $^{21}$ Second, our study was affected by missing data. However, the characteristics of patients with and without missing data were similar. The younger age of children with HADS data is likely to be explained by the fact that, until 2008, children under 14 were not given the HADS questionnaire at baseline. It is possible that children who did not complete the HADS were more likely to be depressed, as studies have shown that people with higher depression scores are less likely to provide complete data ${ }^{22}$ and are less likely to return questionnaires. ${ }^{23}$ Hence, the rates of depression reported here may be underestimated. Third, our study relied upon self-completed questionnaires and we did not conduct diagnostic interviews to identify depression meeting DSM-IV or ICD-10 diagnostic criteria. There is considerable comorbidity between anxiety disorders and depression, ${ }^{8} 24$ and it was not possible to identify the extent of comorbidity or the nature of the primary disorder. Future studies should address this limitation by using diagnostic interviews to quantify the number of children with CFS/ME who fulfil diagnostic criteria for depression and identify children with other comorbid disorders.

\section{Comparison with other studies}

Our study is consistent with previous smaller studies which have demonstrated a higher rate of depression in adolescents with $\mathrm{CFS} / \mathrm{ME}$ than in healthy controls ${ }^{9}{ }^{10}$ and higher depression scores in those with CFS/ME than in the general population. ${ }^{12}$ We found that children with higher HADS depression scores also had higher HADS anxiety scores, which is consistent with previous studies. ${ }^{8}{ }^{24}$ We also found that children with higher HADS depression scores had higher pain scores. This is consistent with reported associations between low back pain and emotional problems in school children ${ }^{25}$ and between musculoskeletal pain and depression in 9-16-year-olds in the Great Smoky Mountain Study. ${ }^{26}$

\section{Meaning of the study and implications for practice}

Depression in CFS/ME is associated with disability, pain, fatigue and anxiety. It is unclear whether there is a causal relationship in either direction. It may be that those with higher levels of disability, pain and fatigue become more depressed as a consequence or that those with depression are more disabled and experience more pain and fatigue as a result. There is a literature on shared vulnerability models for depression and functional somatic symptoms, ${ }^{27}$ but evidence on potential mechanisms is limited. The fact that there is no association between depression and length of illness prior to first assessment 
suggests that risk of depression does not increase the longer a child is ill with CFS/ME. This may constitute evidence against the idea that depression is a consequence of living with the chronic stigmatising condition of $\mathrm{CFS} / \mathrm{ME}^{28}$ As this is a crosssectional study, we do not know whether comorbid depression changes prognosis or whether depression increases the longer a child has CFS/ME after assessment by a specialist service.

High rates of depression in CFS/ME suggest that it is important for clinicians to consider comorbid depression in children with a diagnosis of CFS/ME. Clinicians should be particularly alert for depression in the group of children most severely disabled or affected in terms of fatigue and pain by their CFS/ME, as this group is most likely to have comorbid depression. Specialist CFS/ME teams should have psychologists and psychiatrists working within them so that the depression can be assessed and treated by health professionals who also have a good understanding of CFS/ME. CFS/ME teams should also maintain strong links and clear care pathways with local mental health teams. Cognitive behavioural therapy (CBT) is effective for adolescent depression and CFS/ME $\mathrm{ME}^{291}$ but there are differences in the form of CBT for the two illnesses, and there is very little evidence about effective treatments for those with concurrent CFS/ME and depression. Two randomised controlled trials of CBT for CFS/ME excluded adolescents with major depression and did not assess whether mood improved during treatment. $^{29} 30$ As this comorbidity is common, it is important that effective treatments for CFS/ME with comorbid depression are investigated; however, in the meantime, we would recommend that the usual treatment for depression should be instigated.

\section{CONCLUSIONS}

Depression is a common comorbidity in children and young people with CFS/ME. It is important that clinicians are alert to the possibility of depression and treat it. Further research is required into effective treatments for comorbid CFS/ME and depression.

Acknowledgements We are grateful to all the young people and their families who attend our service.

Contributors HB assisted with interpretation of the data and drafted the article. SMC completed the statistical analysis and assisted with interpretation of the data. EC conceived and designed the study. GL, KR and EC assisted with interpretation of the data. All authors critically revised the manuscript for important intellectual content and approved the final version to be published. EC is guarantor.

Funding This data collection was funded for service evaluation by the Linbury Trust and supported by additional funding from Action for ME.

Competing interests EC is medical advisor to the Association of Young people with ME (AYME) and the Sussex and Kent ME/CFS Society. The work is produced by EC under the terms of a Clinician Scientist Award issued by the NIHR. The views expressed in this publication are those of the author(s) and not necessarily those of the NHS, the National Institute for Health Research or the Department of Health.

Ethics approval The local Research and Ethics committee decided that the collection and analysis of this data were service evaluation and as such did not require an ethical review by a National Health Service Research Ethics Committee or approval from the National Health Service Research \& Development office (07/Q2006/48).

Provenance and peer review Not commissioned; externally peer reviewed.

\section{REFERENCES}

1 Chalder T, Goodman R, Wessely $\mathrm{S}$, et al. Epidemiology of chronic fatigue syndrome and self reported myalgic encephalomyelitis in 5-15 year olds: cross sectional study. BMJ 2003:327:654-5.

2 Jones JF, Nisenbaum R, Solomon L, et al. Chronic fatigue syndrome and other fatiguing illnesses in adolescents: a population-based study. J Adolesc Health 2004;35:34-40.
3 Rimes KA, Goodman R, Hotopf $M$, et al. Incidence, prognosis, and risk factors for fatigue and chronic fatigue syndrome in adolescents: a prospective community study. Pediatrics 2007:119:e603-9.

4 Crawley EM, Emond AM, Sterne JA. Unidentified Chronic Fatigue Syndrome/myalgic encephalomyelitis (CFS/ME) is a major cause of school absence: surveillance outcomes from school-based clinics. BMJ Open 2011;1:e000252.

5 Rangel L, Garralda ME, Levin M, et al. The course of severe chronic fatigue syndrome in childhood. J R Soc Med 2000;93:129-34.

6 NICE. Chronic fatigue syndrome/Myalgic encephalomyelitis (or encephalopathy); diagnosis and management. CG53. National Institute for Health and Clinical Excellence (NICE), 2007

7 Royal College of Paediatrics and Child Health. Evidence based guideline for the management of CFS/ME (chronic fatigue syndrome/myalgic encephalopathy) in children and young people. London, UK: Royal College of General Practitioners, 2004.

8 Ford T, Goodman R, Meltzer H. The British child and adolescent mental health survey 1999: The prevalence of DSM-IV disorders. J Am Acad Child Adolesc Psychiatry 2003;42:1203-11.

9 Carter BD, Edwards JF, Kronenberger WG, et al. Case control study of chronic fatigue in pediatric patients. Pediatrics 1995;95:179-86.

10 Rangel L, Garralda ME, Hall A, et al. Psychiatric adjustment in chronic fatigue syndrome of childhood and in juvenile idiopathic arthritis. Psychol Med 2003;33:289-97.

11 Garralda E, Rangel L, Levin M, et al. Psychiatric adjustment in adolescents with a history of chronic fatigue syndrome. J Am Acad Child Adolesc Psychiatry 1999;38:1515-21.

12 Van Middendorp H, Geenen R, Kuis W, et al. Psychological adjustment of adolescent girls with chronic fatigue syndrome. Pediatrics 2001;107:E35.

13 Smith MS, Martin-Herz SP, Womack WM, et al. Comparative study of anxiety, depression, somatization, functional disability, and illness attribution in adolescents with chronic fatigue or migraine. Pediatrics 2003;111(4 Pt 1):e376-81.

14 White D, Leach C, Sims R, et al. Validation of the Hospital Anxiety and Depression Scale for use with adolescents. Br J Psychiatry 1999;175:452-4.

15 Zigmond AS, Snaith RP. The hospital anxiety and depression scale. Acta Psychiatr Scand 1983;67:361-70.

16 Deale A, Wessely S. Patients' perceptions of medical care in chronic fatigue syndrome. Social Sci Med 2001;52:1859-64.

17 Spence SH. A measure of anxiety symptoms among children. Behav Res Ther 1998;36:545-66.

18 Chalder T, Berelowitz G, Pawlikowska T, et al. Development of a fatigue scale. J Psychosom Res 1993;37:147-53.

19 Garratt AM, Ruta DA, Abdalla MI, et al. The SF-36 Health Survey Questionnaire - an outcome measure suitable for routine use within the NHS. BMJ 1993:306:1440-4.

20 Jorngarden A, Wettergen L, von Essen L. Measuring health-related quality of life in adolescents and young adults: Swedish normative data for the SF-36 and the HADS, and the influence of age, gender, and method of administration. Health Qual Life Outcomes 2006:4:91.

21 Richards J, Turk J, White $S$. Children and adolescents with chronic fatigue syndrome in non-specialist settings: beliefs, functional impairment and psychiatric disturbance. Eur Child Adolesc Psychiatry 2005;14:310-18.

22 Ramchandani PG, Stein A, O'Connor TG, et al. Depression in men in the postnatal period and later child psychopathology: a population cohort study. J Am Acad Child Adolesc Psychiatry 2008;47:390-8.

23 Heron J, O'Connor TG, Evans J, et al. The course of anxiety and depression through pregnancy and the postpartum in a community sample. J Affect Disord 2004;80:65-73.

24 Costello EJ, Mustillo S, Erkanli A, et al. Prevalence and development of psychiatric disorders in childhood and adolescence. Archives of General Psychiatry 2003;60:837-44.

25 Watson KD, Papageorgiou AC, Jones GT, et al. Low back pain in schoolchildren: the role of mechanical and psychosocial factors. Arch Dis Child 2003;88:12-17.

26 Egger HL, Costello EJ, Erkanli A, et al. Somatic complaints and psychopathology in children and adolescents: stomach aches, musculoskeletal pains, and headaches. J Am Acad Child Adolesc Psychiatry 1999;38:852-60.

27 Campo JV. Annual research review: functional somatic symptoms and associated anxiety and depression-developmental psychopathology in pediatric practice. J Child Psychol Psychiatry 2012;53:575-92.

28 Bould $\mathrm{H}$, Lewis $\mathrm{G}$, Emond $\mathrm{A}$, et al. Depression and anxiety in children with CFS/ME: cause or effect? Arch Dis Child 2011;96:211-14.

29 Chalder T, Deary V, Husain K, et al. Family-focused cognitive behaviour therapy versus psycho-education for chronic fatigue syndrome in 11- to 18-year-olds: a randomized controlled treatment trial. Psychol Med 2010;40:1269-79.

30 Stulemeijer M, de Jong LW, Fiselier TJ, et al. Cognitive behaviour therapy for adolescents with chronic fatigue syndrome: randomised controlled trial. BMJ 2005;330:14.

31 Lloyd S, Chalder T, Rimes KA. Family-focused cognitive behaviour therapy versus psycho-education for adolescents with chronic fatigue syndrome: long-term follow-up of an RCT. Behav Res Ther 2012;50:719-25. 


\section{Correction: Depression in paediatric chronic fatigue syndrome}

Bould H, Collin SM, Lewis G, et al. Depression in paediatric chronic fatigue syndrome. Arch Dis Child 2013;98:425-8.

The Editor of the journal has agreed to a request from the authors that the ethics statement should be changed to improve its clarity. This request was received in October 2019. Due to an internal miscommunication at the publisher the correction was not made. The publisher apologises for this oversight. The new statement is as follows:

'The North Somerset \& South Bristol Research Ethics Committee decided that the collection and analysis of clinical data collected by the regional specialist service for service evaluation did not require ethical review by a National Health Service Research Ethics Committee or approval from the National Health Service Research \& Development Office (07/Q2006/48).'

(C) Author(s) (or their employer(s)) 2022. No commercial re-use. See rights and permissions. Published by BMJ.

Arch Dis Child 2022;107:e33. doi:10.1136/archdischild-2012-303396.corr1

(A) Check for updates 